\title{
Advances in endocrinology
}

\author{
John P Monson
}

\section{Introduction}

To those who do not know me I should begin with a brief but relevant biography to establish my frame of reference for this topic. My personal experience in clinical endocrinology spans a period of 32 years commencing with registrar training on the St George's Hospital rotation in 1975. Prior to this I had some general introduction to endocrinology during my undergraduate training at Guy's Hospital and in the context of general medical training appointments but it was undoubtedly my exposure to diabetes and endocrinology while working for Dr GS Spathis in 1975 which confirmed my future career intentions. Subsequent important influences included Professors TRE Pilkington and Peter Richards at St George's, and Professor RD Cohen, Dr AS Mason and Dr BJ Boucher at the London Hospital. In 1982 I was appointed Senior Lecturer and Honorary Consultant at the the London and Oldchurch Hospitals, later becoming Reader in Metabolism and Endocrinology before moving to St Bartholomew's Hospital in 1995 as Reader in Medicine. I was appointed Professor of Clinical Endocrinology at Barts and The London in 2000 and became Lead Endocrinologist at St Bartholomew's in 2001 on the retirement of Professor GM Besser. I retired from my university appointment in 2006 but have continued research in an emeritus capacity and remain active in private clinical practice at the London Clinic Centre for Endocrinology.

In order to examine the extensive changes in clinical endocrinology which have occurred during the past 30 to 40 years I will deal separately with changes in working practice, advances in clinical biochemistry, the impact of advanced imaging modalities, the impact of molecular genetics, surgical developments and important pharmacological advances.

\section{Working practices in clinical endocrinology}

In common with other medical specialties the past 30 years have seen major changes in the conduct of routine endocrinological practice. These changes have been determined by several factors including the advance of specialisation and the changing role of the general physician. Whereas it was previously the norm for generalists to see and treat common endocrine disorders, the climate has progressively changed with the majority of generalists majoring in their own specialties for all but their contribution to

\footnotetext{
John P Monson, Emeritus Professor of Clinical Endocrinology,

Centre for Endocrinology, William Harvey Research Institute,

St Bartholomew's and the Royal London School of Medicine,

Queen Mary, University of London
}

undifferentiated acute medicine. Throughout the 1970s and 1980s, increasing recognition of the importance of optimum diabetes care led to more district general hospital consultant appointments in diabetes and endocrinology with the added benefit of provision of local endocrine services. Paradoxically the recent shift away from secondary diabetes care and the reduction in investment in relevant hospital services has had the unfortunate consequence of threatening availability of local expertise in endocrinology. This latter phenomenon illustrates very clearly the problem of recognition of the importance of endocrinology among purchasers of healthcare.

Highly specialised endocrine services have traditionally been provided by tertiary referral departments in a teaching hospital setting supported by dedicated investigation units staffed by specialist nurses. ${ }^{1}$ Interestingly, such departments have frequently been embedded within traditional professorial medical units, reflecting the academic credentials of the specialty and its clear relationship with general (internal) medicine. The demise of the generic academic department of medicine has to some extent led to a redefinition of academic endocrinology as a stand alone laboratory-based discipline. There has been understandable anxiety that, as a consequence of well-recognised academic imperatives, fuelled by the research assessment exercise, clinical endocrinology and clinical endocrinology research would become separated from the academic laboratory and subject to the vagaries and short-term imperatives of the NHS maelstrom. This phenomenon, which has been evident over approximately 10 years, may be resolved by belated recognitions of the importance of 'translational' research. The perennial problem faced by clinical endocrinologists is to convince an audience of purchasers accustomed to the costing of procedure-based medicine that optimum treatment of hormonal disorders requires a specialist approach.

\section{Advances in clinical biochemistry}

It would be difficult to overestimate the contribution of advances in hormonal assay techniques to clinical endocrine practice. The techniques of in vivo and in vitro bioassay for peptide hormones, measurement of protein-bound iodine as an index of thyroid function and chromatography and fluorimetry for steroids were the mainstay of endocrine investigation until the advent of immunoassays some 40 years ago. Since then refinements in methodology have progressed from basic radioimmunoassay to immunoradiometric assays, free hormone assays and ELISA. ${ }^{2}$ A direct consequence of these developments was a rapid increase in understanding of pathophysiology of complex endocrinopathies, increased precision in 
diagnosis and enhancement of patient care. Wider availability of specific hormonal measurements, initially through supraregional assay services and later as a result of improvements in automation of assays, brought these important tools to endocrinologists working outside traditional teaching centres. Taking the most common endocrine scenario of thyroid dysfunction, the impact of reliable assays for thyroid hormones and thyroidstimulating hormone (TSH) have been of incalculable value in all clinical settings. Inevitably, advances in assay precision have prompted new questions because of the potential to diagnose so-called 'subclinical' disorders for which the appropriate clinical strategy remains unclear.

\section{The impact of advanced imaging modalities}

Such is the importance of specific imaging modalities in the diagnosis of endocrine disease that the young endocrinologist of today may have difficulty envisaging an era prior to computerised tomography (CT) and magnetic resonance imaging (MRI). Imaging of the abdomen took a major leap forward with the development of precise ultrasound techniques over 30 years ago. Taking one example, prior to the advent of ultrasound, imaging of the adrenal glands depended on achieving X-ray contrast by the technique of peri-renal air insufflation, an uncomfortable and imprecise procedure. The diagnosis of pituitary lesions was equally difficult and depended on air encephalography, supported by plain X-ray polytomography. Much changed with the advent of CT imaging in the early 1970s; the earliest models were inadequate for anything other than large pituitary lesions but proved very useful in the definition of adrenal pathology. Precise imaging of intra-abdominal neuroendocrine malignancy awaited higher resolution CT imaging and awareness of the importance of arterial phase contrast imaging for the detection of hepatic neuroendocrine metastases. Lung windowed, thin section CT scanning remains the imaging modality of choice for the identification of pulmonary lesions including small bronchial carcinoid tumours. ${ }^{3}$

MRI, with increasing precision and speed of image acquisition, has revolutionised endocrine imaging. In relation to the pituitary, MRI permitted more precise definition of parasellar structures and small intrasellar pituitary adenomas. ${ }^{4}$ It also proved invaluable in the evaluation of pancreatic neuroendocrine tumours and the identification of phaeochromocytoma and paraganglioma. One of its major strengths was in permitting long-term repeated surveillance observation without radiation risk. This latter benefit has been a major determinant of the current conservative approach to residual pituitary tumour following incomplete surgical resection.

The precision of anatomical imaging now possible has resulted in the frequent identification of incidental lesions. Some (eg adrenal lesions) are of uncertain significance but may result in unforeseen investigation requirements and increased patient anxiety. We should therefore acknowledge that, in some instances, the sophistication of imaging techniques now exceeds our understanding of the natural history of some disorders.
Another important point, emerging clearly in endocrine practice, is that cross-sectional imaging techniques do not confirm pathology, but rather serve to localise an established diagnosis. Indeed, the early application of imaging for a diagnosis which is suspected but not confirmed may prove seriously misleading, not least because of the high prevalence of incidental non-functioning, benign lesions in the adrenal, thyroid and pituitary.

The ability to add a functional element to an imaging study has been a further important advance achieved by application of angiography techniques and selective venous sampling. Examples include selective arterial calcium stimulation with hepatic venous sampling for the investigation of islet cell tumours, selective venous sampling for adrenal and parathyroid lesions and bilateral, simultaneous petrosal sinus sampling for the confirmation of adrenocorticotropic hormone (ACTH)-dependent Cushing's disease. ${ }^{5}$

In parallel with advances in cross-sectional imaging techniques there has been increased sophistication in the application of nuclear medicine techniques for diagnosis and therapy. Isotope technology has longstanding proven value in the investigation and treatment of thyroid disease but more recent developments have included labelled MIBI parathyroid scanning, labelled octreotide scanning for neuroendocrine tumours and iodine-123 and I-131 labelled MIBG imaging and therapy for phaeochromocytoma and metastatic neuroendocrine tumours. ${ }^{6}$

\section{The impact of molecular genetics}

The extensive advances in our understanding of the molecular basis of disease have not as yet been translated into direct genetic therapies for endocrine disease although a number of candidates are under investigation. However, important benefits from genetic and molecular investigation have been evident in the prediction of disease (eg diabetes mellitus); understanding mechanisms of disease (eg constitutive activation of Gs signalling in some cases of acromegaly) and in facilitating clinical management of inherited neoplasia syndromes (eg multiple endocrine neoplasia types 1,2A and 2B and von Hippel-Lindau syndrome). Direct mutation analysis has proven particularly valuable in the early management of pre-malignant disease (especially for medullary thyroid neoplasia in MEN2A) and in eliminating mutation-negative individuals and their offspring from the need for further follow-up.

\section{Surgical developments}

In considering the impact of surgery on the treatment of endocrine disorders it would be seriously remiss to ignore the seminal contributions of Harvey Cushing who, by virtue of meticulous clinical observation and surgical excellence, added hugely to our understanding of functioning pituitary disease. He had also excelled in thyroid surgery as a resident at Johns Hopkins University Hospital, Baltimore, MD. Indeed, Cushing can arguably be regarded as one of the founders of modern clinical endocrinology. In the ensuing years transsphenoidal surgery, as performed but later abandoned by Cushing, was not 
pursued largely because of disappointing results and complications. The advent of the operating microscope changed things considerably so that from the late 1960s onwards the technique of transsphenoidal surgery underwent a renaissance and eventually became the procedure of choice for pituitary tumours with control of mass effects and cure of corticotroph and somatotroph lesions, particularly those confined within the sella turcica. Later developments, particularly the advent of transnasal endoscopic techniques increased the surgeon's therapeutic armamentarium. The last 15 years have seen increasing recognition of the importance of specialisation in pituitary surgery with clear evidence of improved results as a direct result of surgical experience.

Surgical treatment of thyroid and parathyroid disease was traditionally the province of the interested general surgeon. However, audit of results has left little doubt as to the value of increased specialisation in this area, particularly in terms of avoidance of surgical complications, reducing the rate of re-exploration for hyperparathyroidism and optimising the treatment of thyroid malignancy. The need for specialisation in the surgical treatment of adrenal, pancreatic and other neuroendocrine disorders has been long recognised. However, the development of techniques for more extensive surgery in the treatment of intra-abdominal malignant disease, including partial hepatic resections, has provided an important role for the hepatobiliary surgeon in recent years and has expanded the contribution of surgery in cytoreduction of metastatic endocrine tumours.

\section{Physicians and surgeons}

We now work in the era of the multidisciplinary team. Valuable though these structures are, it would be a mistake to imagine that they are particularly new. They have, like the majority of advances in clinical management process, been born out of the pioneering efforts and experience of individual physicians and surgeons. However, now and again a particular event will have a catalytic effect on clinical attitudes and cooperation which may previously have been low key or deemed to be of borderline importance. Such indeed was the impact of medical treatment of lactotroph macroadenomas with dopamine agonists in the 1970s. ${ }^{7}$ This development, which proved capable of dramatically shrinking these tumours and restoring vision was a revolution in clinical management while simultaneously emphasising the importance of primary evaluation of all patients with pituitary lesions by a clinical endocrinologist. The consequence was improvement in integrated care of patients with pituitary disease.

\section{Pharmacological advances}

Replacement therapies for thyroid, adrenal and pituitary failure have continued to be refined over the past three decades by virtue of the availability of recombinant hormone production and the development of specific assays permitting optimisation of dose quantity and treatment schedules. Recognition of the impact of dopamine agonist therapy, initially bromocriptine, on prolactin hypersecretion provided the first effective medical therapy for a specific functioning pituitary tumour. ${ }^{7}$ The subsequent demonstration of a growth hormone lowering effect of bromocriptine in acromegaly was rightly regarded as a breakthrough despite the relatively modest improvement in many patients. Some 10 years after the initial publications documenting benefit from bromocriptine therapy, the medical treatment of functioning endocrine tumours was advanced by the development of somatostatin analogues which demonstrated potent inhibitory effects on secretion by the majority of somatotroph adenomas and gastrointestinal neuroendocrine tumours. The medical treatment of acromegaly has been further advanced by the development of modified growth hormone molecules capable of blocking the growth hormone receptor and thereby mitigating the adverse effects of excess growth hormone at a tissue level. ${ }^{8}$

\section{Conclusions}

This synopsis of advances in endocrinology spanning 30 to 40 years provides some indication of the extensive investigational and therapeutic tools now available to the practicing endocrinologist. It is nonetheless important to recognise that investigations, no matter how refined, cannot replace skilled and detailed clinical assessment but rather provide additional support. This latter point bears repeating, particularly to those who mistakenly imagine that technology may render traditional clinical practice obsolete.

\section{References}

1 Monson JP. Endocrine units. In: Wass JAH, Shalet SM (eds). Oxford textbook of endocrinology. Oxford: Oxford University Press, 2002: $61-4$.

2 Price CP, Newman DJ (eds). Principles and practice of immunoassay. London: Macmillan, 1997.

3 Reznek RH, Dacie JE. General radiology of endocrine disease. In: Besser GM, Thorner MO (eds). Comprehensive clinical endocrinology. New York: Mosby, 2002:633-78.

4 Buchfelder M, Nistor R, Fahlbusch R, Huk WJ. The accuracy of CT and MR evaluation of the sella turcica for detection of adrenocorticotropic hormone-secreting adenomas in Cushing's disease. Am J Neuroradiol 1992:14:1183-90.

5 Oldfield EH, Doppman JL, Nieman LK et al. Petrosal sinus sampling with and without corticotrophin-releasing hormone for the differential diagnosis of Cushing's syndrome. N Engl J Med 1991;325:897-905.

6 Britton KE. Nuclear medicine in endocrine disease. In: Besser GM, Thorner MO (eds). Comprehensive clinical endocrinology. New York: Mosby, 2002:679-94.

7 Thorner MO, McNeilly AS, Hagan C, Besser GM. Long-term treatment of galactorrhoea and hypogonadism with bromocriptine. BMJ 1974;2:419-22.

8 Trainer PJ, Drake WM, Katznelson L et al. Treatment of acromegaly with the growth hormone-receptor antagonist pegvisomant. $\mathrm{N} \mathrm{Engl} \mathrm{J}$ Med 2000;342:1171-7.

Address for correspondence: Professor JP Monson, London Clinic Centre for Endocrinology, 5 Devonshire Place, London W1G 6HL. Email: Johnmonson@aol.com 